\title{
Nitrate respiration occurs throughout the depth of mucoid and non-mucoid Pseudomonas aeruginosa submerged agar colony biofilms including the oxic zone
}

\section{Paul Stoodley ( $\sim$ Paul.Stoodley@osumc.edu )}

The Ohio State University

\section{Carsten Ulrich Schwermer}

Norwegian Institute for Water Research (NIVA)

Dirk deBeer

Max Planck Institute for Marine Microbiology (MPI)

\section{Research Article}

Keywords:

Posted Date: February 3rd, 2022

DOI: https://doi.org/10.21203/rs.3.rs-1254260/v1

License: (c) (1) This work is licensed under a Creative Commons Attribution 4.0 International License. Read Full License 


\section{Abstract}

Pseudomonas aeruginosa is an opportunistic pathogen and well characterized biofilm former. $P$. aeruginosa forms strong oxygen gradients inside biofilms due to rapid oxygen respiration in the top layers and the poor solubility of oxygen coupled with diffusion limited transport. Transcriptomic evidence from in vitro and ex vivo sampling suggests that denitrification is occurring in biofilms in ostensibly oxic environments. It is hypothesized that in the presence of nitrate there is stratification with aerobic respiration occurring in the outer oxic layer and denitrification in the lower anoxic zone. We used submerged agar colony biofilms grown from mucoid (FRD1) and non-mucoid (PAO1) strains to simultaneously measure depth microprofiles of oxygen and nitrous oxide in the same colony with microelectrodes. Oxygen respiration occurred at the top of the colony as expected but denitrification occurred throughout the entire depth, even in the oxic region. Local denitrification rates were highly variable suggesting heterogenous metabolic activity within the colony. We also assessed the short-term influence of tobramycin on aerobic respiration within a PAO1 colony. Although there was an immediate reduction in respiration it was never completely arrested over $2 \mathrm{hr}$. On tobramycin removal the oxygen gradient steadily reestablished, demonstrating immediate recovery of metabolic activity.

\section{Introduction}

Pseudomonas aeruginosa is a Gram-negative opportunistic pathogen and is a well characterized biofilm former. Biofilm formation by this organism is a recognized virulence factor in chronic infections such as occurring in the cystic fibrosis (CF) lung ${ }^{1}$ and unhealing wounds ${ }^{2}$. Anaerobic nitrate $\left(\mathrm{NO}_{3}{ }^{-}\right)$respiration (denitrification), even in an ostensibly physiologically aerobic environment, such as the lung, has been found to be a relevant factor in the pathogenesis of this organism ${ }^{3,4}$. It is thought that hypoxic conditions in the mucus of the CF lung are caused by greater consumption of oxygen by the underlying CF epithelial cells ${ }^{5}$. However, it is well established that biofilms formed from aerobic or facultative aerobes, such as $P$. aeruginosa, can form strong oxygen gradients due to the rate of aerobic respiration near the surface of the biofilm consuming oxygen at a greater rate than oxygen can diffuse in from the overlying fluid ${ }^{6}$. Expression levels of genes of enzymes involved in denitrification [i.e., $2 \mathrm{NO}_{3}{ }^{-} \rightarrow 2 \mathrm{NO}_{2}{ }^{-} \rightarrow 2 \mathrm{NO}^{-} \rightarrow \mathrm{N}_{2} \mathrm{O} \rightarrow$ $\mathrm{N}_{2}$ by nitrate reductase (NAR), nitrite reductase (NIR), nitrous oxide reductase (NOR), and nitrous oxide reductase (NOS) ${ }^{7}$ were found to be upexpressed in anaerobically grown $P$. aeruginosa biofilms. Also, the outer membrane protein OprF, which is associated with anaerobic growth, was found in biofilms collected from P.aeruginosa infected clinical samples of $\mathrm{CF}_{\text {sputum }}{ }^{8}$, providing evidence for anaerobic growth in the CF lung. In addition, denitrification in $P$. aeruginosa was demonstrated in vitro under hypoxia and physiologically relevant $\mathrm{NO}_{3}{ }^{-}$concentrations $(400 \mu \mathrm{M})$ of the CF lung by RT-PCR, $\mathrm{N}_{2} \mathrm{O}$ microelectrodes, GFP reporter constructs and proteomic ${ }^{4,9}$. While these studies provide compelling evidence that denitrification metabolism by $P$. aeruginosa occurs under hypoxia and are likely occurring in the CF lung, they do not provide data on spatial distribution of anaerobic activity within the biofilm. It is generally thought that the outside layer of the biofilm adjacent to the oxygenated fluid would be dominated by 
aerobic respiration, while the less energetically favorable anaerobic respiration or fermentation would occur in the interior of the biofilm, resulting in species and phenotypic stratification in a mixed species biofilm ${ }^{10}$. Unlike oxygen, which is sparingly soluble in water (i.e. $8.3 \mathrm{mg} / \mathrm{L}$, at $25^{\circ} \mathrm{C}, 0.9 \%$ salinity and 1 bar), $\mathrm{NO}_{3}{ }^{-}$as an alternative electron acceptor is over a thousand times more soluble and thus may be expected to fully penetrate the biofilm. We hypothesized that in the presence of both oxygen and $\mathrm{NO}_{3}$; aerobic respiration would occur in the upper layer of the biofilm and that denitrification would occur primarily below this layer in the anoxic region created by oxygen depletion. Previously microelectrodes for different ions and molecules have been used to create concentration with depth profiles in biofilms to understand the distribution of different metabolic processes and to infer the stratification of different physiological species in biofilms to assess community interactions and local and overall metabolic activities $^{11}$. Here we used a simple in vitro model to simulate biofilms growing on soft surfaces where colonies of mucoid (FRD1) and non-mucoid (PA01) P. aeruginosa strains were grown on agar plates and submerged in a glass aquarium reservoir. The model was specifically designed to allow the simultaneous measurement of vertical high-resolution microprofiles of dissolved oxygen and nitrous oxide $\left(\mathrm{N}_{2} \mathrm{O}\right)$ after spiking with $\mathrm{NO}_{3}{ }^{-}$by microelectrodes, which were introduced at the same depth of the biofilm, angled in from different sides. The corresponding $\mathrm{NO}_{3}{ }^{-}$microprofile was calculated from the stoichiometry of $\mathrm{N}_{2} \mathrm{O}$ production.

\section{Materials And Methods}

\section{Bacterial strains, growth conditions and experimental set-up}

We used $P$. aeruginosa PA01, a non-mucoid wound isolate ${ }^{12}$, and FRD1 a mucoid cystic fibrosis isolate ${ }^{13}$. Frozen stock cultures were streaked out on brain heart infusion agar BHI (Difco 241830) and incubated for $24 \mathrm{hr}$ at room temperature $\left(24^{\circ} \mathrm{C}\right)$ for three days. $\mathrm{BHI}$ has been noted to suppress mucoidy in $P$. aeruginosa $^{14}$, allowing distinct colonies of FRD1 to present on the agar. The average thickness of the biofilm colonies on a dedicated set of plates was measured daily by microscopy by focusing on the top of the colonies and the adjacent bare agar and noting the distance travelled using the scale on the focusing knob (Figure 1). The plates were then transferred to a square glass sided (approximately $14 \times 14$ $x 7 \mathrm{~cm}$ in dimensions) aquarium reservoir which was gently filled with $0.75 \mathrm{~L}$ of minimal salts media (MSM supplemented with glucose in g/L: $0.7 \mathrm{~K}_{2} \mathrm{HPO}_{4}, 0.3 \mathrm{KH}_{2} \mathrm{PO}_{4}, 0.01 \mathrm{NH}_{4} \mathrm{SO}_{4} \mathrm{MgSO}_{4}$ * $7 \mathrm{H}_{2} \mathrm{O}, 0.4$ glucose, all from Sigma-Aldrich) to submerge the biofilm. The $\mathrm{pH}$ was adjusted to 7.3 with $\mathrm{NaOH}$. The Petri plate was weighed down by wrapping with a flexible plastic-coated lead flask weight. A recirculating pump was used to generate water flow to allow mixing in the reservoir and air was introduced through an air stone to maintain oxygen saturation in the water phase. Floating balls (Allplas, Capricorn Chemicals Corp., Secaucus, NY) covered the water surface to minimize gas exchange with the atmosphere. When the MSM was added to the reservoir there was no immediate visible disruption to the colonies (Figure 1C, D); however, in preliminary experiments after 3 or 4 hours we found there was dissolution of the colonies 
with a concomitant clouding of the media, thus we performed our experiments within this limited time frame.

\section{Microelectrodes and calibration.}

Dissolved oxygen (DO) and nitrous oxide $\left(\mathrm{N}_{2} \mathrm{O}\right)$ microelectrodes with tip diameters of approximately 10 $\mu \mathrm{m}$ were fabricated at the Max Planck Institute for Marine Microbiology as previously described ${ }^{15}, 16$. The build-up of $\mathrm{N}_{2} \mathrm{O}$ on the addition of $\mathrm{NO}_{3}{ }^{-}$is indicative of denitrification according to the reaction series. The microelectrodes were connected to a picoamperemeter. The amplifier converts atmospheric pressure (pA) to millivolt $(\mathrm{mV})$ which were recorded by a data acquisition system (model DAQCard Al16XE50; National Instruments, Austin, TX) on a laptop. DO electrodes were calibrated in saturated (air sparged) and anoxic ( $\mathrm{N}_{2}$ sparged) phosphate buffered water (MSM). The $\mathrm{N}_{2} \mathrm{O}$ electrodes were calibrated with MSM solutions at $0,100,200,300,400 \mu \mathrm{M} \mathrm{N} \mathrm{N}_{2} \mathrm{O}$ by first saturating the MSM with $\mathrm{N}_{2} \mathrm{O}$ gas and then by dilution. Since the $\mathrm{N}_{2} \mathrm{O}$ in solution slowly escape to the atmosphere, the concentration can only be considered constant for a few minutes, thus the calibration was performed quickly. There is a linear relationship between measured pA and $\mathrm{N}_{2} \mathrm{O}$ concentration (Supplemental Figure 1). Some bacteria only reduce $\mathrm{NO}_{3}{ }^{-}$to $\mathrm{N}_{2} \mathrm{O}$. However, if there is complete reduction to $\mathrm{N}_{2}$ gas, $\mathrm{N}_{2} \mathrm{O}$ is consumed and consequently denitrification activity may be underestimated. Pseudomonas aeruginosa is not known to produce $\mathrm{N}_{2}$ gas but as a control to check this with our strains, acetylene ( $10 \%$ of a saturated solution in the MSM achieved by sparging with pure acetylene gas), which prevents the reduction of $\mathrm{N}_{2} \mathrm{O}$ to $\mathrm{N}_{2}{ }^{17}$, was added after a $\mathrm{NO}_{3}{ }^{-}$spike $(1.2 \mu \mathrm{M})$ and no difference in $\mathrm{N}_{2} \mathrm{O}$ concentration was seen (Supplemental Figure 2).

\section{Microelectrode measurements}

The DO and $\mathrm{N}_{2} \mathrm{O}$ microelectrodes were inserted into the reservoir through a circular "window" fabricated from the top of a plastic wide mouth container using a micromanipulator (model MM33; Maerzhaeuser, Wetzlar, Germany) with a motor-controlled z-axis stepper (model VT-80; Micos, Eschbach, Germany). Positioning during vertical profiling and data acquisition were controlled by using custom-made software. The electrodes were simultaneously lowered onto the surface of the same colony biofilm while being observed with a dissecting microscope under illumination by a gooseneck lamp (Schott). The approximate surface of the colony biofilm was identified by the point at which the tips of the microelectrodes and their shadows cast by the gooseneck lamp co-localized (Figure 2). A more precise identification of the colony-bulk fluid interface was made by noting a rapid response of the DO microelectrode, which was between 25 and $50 \mu \mathrm{m}$ deeper than the visual estimate. The surface of the colony biofilm was designated as depth $0 \mu \mathrm{m}$. Distances above the interface in the bulk fluid were designated as negative and below into the colony biofilm as positive. Profiles of $\mathrm{DO}$ and $\mathrm{N}_{2} \mathrm{O}$ outside and within the colonies were generated generally from $500 \mu \mathrm{m}$ above the surface to a depth of $500 \mu \mathrm{m}$ into the biofilm at $25 \mu \mathrm{m}$ steps. The stepper motor travel step distance to achieve $25 \mu \mathrm{m}$ steps at the tips of the microelectrodes was calculated from the angle of insertion by simple geometry. The reservoir system 
and microelectrodes entering a colony biofilm are shown in Figure 2. Denitrification was induced by spiking the reservoir with a hundred times concentrated $10 \mathrm{~g} / \mathrm{L} \mathrm{NaNO}_{3}$-stock solution to achieve a final concentration of $0.1 \mathrm{~g} / \mathrm{L}(1.2 \mathrm{mM})$ of $\mathrm{NO}_{3}{ }^{-}$. DO and $\mathrm{N}_{2} \mathrm{O}$ profiles were measured repeatedly at the same locations until steady state was reached, which is an important condition for flux calculations. To calculate aerobic respiration and denitrification rates and activities as a function of depth two sets of profiles were measured from different colonies on the same plate. The measurements are therefore suitable to compare these activities in almost the same location but do not provide information on variability between biofilm colonies grown on different plates, or indeed, cannot be used to make conclusions regarding differences between colonies on the same plate.

\section{Short term exposure to tobramycin}

Since anoxic conditions within $P$. aeruginosa biofilms have been associated with tolerance to many antibiotics including aminoglycosides, quinolones and $\beta$-lactams ${ }^{18}$ through either dormancy or the production of phezanines ${ }^{6}$ in the anoxic regions, we assessed the shorth term exposure of tobramycin on aerobic respiration at $100 \mu \mathrm{m}$ depth in a PA01 biofilm colony. A profile between $-800 \mu \mathrm{m}$ to $400 \mu \mathrm{m}$ depth in $20 \mu \mathrm{m}$ steps into the biofilm was taken $1.0 \mathrm{hr}$ prior to tobramycin addition at $10 \mu \mathrm{g} / \mathrm{mL}$ in MSM (approximately 5 times the minimum inhibitory concentration, MIC). The DO microelectrode was then parked at $100 \mu \mathrm{m}$ depth and the influence on aerobic respiration activity measured as decreasing DO concentration, The DO was measured for $1.0 \mathrm{hr}$ to ensure steady state, and then for a further $2.0 \mathrm{hr}$ after tobramycin addition. A second profile was taken at $2.5 \mathrm{hr}$ before exchanging the reservoir with MSM with no antibiotic and measurements continued for a further $2.5 \mathrm{hr}$.

\section{Dissolved oxygen (DO) and $\mathrm{NO}_{3}{ }^{-}$flux calculations}

Since denitrification from $\mathrm{NO}_{3}{ }^{-}$to $\mathrm{N}_{2} \mathrm{O}$ proceeds in a stoichiometry of $1: 1$, we calculated the $\mathrm{NO}_{3}{ }^{-}$ concentration at each level in the profile by subtracting the molar concentration consumed in the production of the measured concentration of $\mathrm{N}_{2} \mathrm{O}$ from the starting $\mathrm{NO}_{3}{ }^{-}$concentration of $1.2 \mathrm{mM}$ in the bulk fluid. We calculated the flux of $\mathrm{DO}$ and $\mathrm{NO}_{3}{ }^{-}$consumption by the colonies as well as a function of depth to determine where aerobic respiration and denitrification were predominantly occurring in the agar colony biofilm. At steady state the diffusive transport of solutes through the diffusive boundary layer $(\mathrm{DBL})$ in the bulk fluid adjacent to the colony is proportional to the concentration gradient in the DBL according to Fick's first law of diffusion: $J=D_{c}(\partial c / \partial x)$, where $J$ is the flux $\left(n m o l ~ c m^{-2} s^{-1}\right), D_{c}$ is the molecular diffusion coefficient $\left(\mathrm{cm}^{2} \mathrm{~s}^{-1}\right)$, and $\partial c / \partial x$, is the concentration gradient $\left(\mathrm{nmol} \mathrm{cm}{ }^{-4}\right)$ at the colony biofilm bulk fluid-interface ${ }^{19,25}$. Assuming a $D_{c}$ in water of $2.36^{*} 10^{-5} \mathrm{~cm}^{2} \mathrm{~s}^{-1}$ for oxygen and of $1.84 * 10^{-5} \mathrm{~cm}^{2} \mathrm{~s}^{-1}$ for $\mathrm{NO}_{3}{ }^{-}$at $24^{\circ} \mathrm{C}^{21,22}$, and correcting for a reduced effective $\mathrm{D}_{\mathrm{c}}\left(\mathrm{D}_{\text {eff }}\right)$ in the biofilm of approximately $58 \%$ and $68 \%$ of that in water as estimated for both $\mathrm{DO}$ and $\mathrm{NO}_{3}{ }^{-}$respectively ${ }^{23}$, areal uptake rates can be calculated that represent the total biofilm by the colony. Local activity of aerobic respiration and denitrification at different depths in the colony biofilm was found from the slope of the profiles within the biofilm colonies using a modified version of Fick's second law of diffusion (assuming 
diffusion is the dominant transport process within the biofilm): $D_{\text {eff }}\left(\partial^{2} c / \partial x^{2}\right)=r$, where $\partial^{2} c / \partial x^{2}$ is the concentration gradient between each step and $r$ is the local conversion rate $\left(\mathrm{mol} \mathrm{cm}^{-3} \mathrm{~s}^{-1}\right)^{24}$. The overall fluxes of $\mathrm{DO}$ and $\mathrm{NO}_{3}{ }^{-}$into the colonies were calculated from the concentration - distance slope of the

first three points at the surface $(0-50 \mu \mathrm{m})$ and multiplying by the assumed $D_{\text {eff }}$ as previously described ${ }^{20}$. While we produced many profiles showing similar trends, we chose overall activity fluxes that were calculated from two representative profiles from each strain taken at different biofilm colonies from the same plate. Therefore, these values can be used to compare the relative local uptake rates of $\mathrm{NO}_{3}{ }^{-}$and DO associated with those particular colonies, but do not account for biofilm heterogeneity and are therefore not suitable for the calculation of average fluxes over the total biofilm surface as previously discussed ${ }^{20}$. Similarly, these data are not statistically robust enough to draw strong conclusions regarding differences of fluxes between the two strains.

\section{Results}

After $72 \mathrm{hr}$ incubation at room temperature, both PA01 and FRD1 colonies had grown to approximately $400 \mu \mathrm{m}$ thickness (Figure 1). In both PA01 and FRD1 sharp DO gradients developed at the colony biofilm bulk liquid interface and the colonies became anoxic at approximately $100 \mu \mathrm{m}$ (PA01) and $150 \mu \mathrm{m}$ (FRD1) depths (Figure 3A, B), with the maximum $\mathrm{N}_{2} \mathrm{O}$ concentration at steady state being at approximately $200 \mu \mathrm{m}$ (PA01) and $250 \mu \mathrm{m}$ (FRD1) depth (Figure 3C, D). Local activity profiles showed that the greatest /DO consumption was occurring in the surface layers of the biofilm colonies and steadily decreased with depth until there was little or no DO respiration $\leq 100 \mu \mathrm{m}$ (Figure 4A, B). However, the calculated $\mathrm{NO}_{3}{ }^{-}$consumption showed that denitrification was more uniform throughout the depth of the colony and was occurring in the surface layers of the biofilm colonies in the oxic zone (Fig. 4C, D). For both strains the overall consumption rate of $\mathrm{DO}$ was much greater than that of $\mathrm{NO}_{3}{ }^{-}$by a factor of 3 and 22 for PA01 and FRD1, respectively (Figure 5).

The response of tobramycin addition colony biofilms was investigated by means of a DO microsensor positioned in the aerobic respiration zone at $100 \mu \mathrm{m}$ depths inside the colony. Figure 6A shows that during the first hour after starting the experiment, the DO saturation was in steady state and below $10 \%$, indicative for an actively ongoing aerobic respiration. Upon addition of tobramycin, the DO saturation rapidly increased from $<10 \%$ to approximately $50 \%$ within $0.5 \mathrm{hr}$ as less oxygen was consumed by overlying cells allowing greater penetration into the biofilm A steady stated in air saturation at ca. $55 \%$ saturation was reached within ca. $1 \mathrm{hr}$ after tobramycin addition. However, after tobramycin was removed by changing the medium the DO began to steadily fall as the gradient was restablished, suggesting that aerobic respiration rapidly recovered.

In addition to the dynamics in aerobic respiration as response to tobramycin addition, Figure 6B shows the spatial change of the aerobic respiration zone inside the PAO1 colony biofilms. In the absence of tobramycin (Figure 6B, profile a'), the presence of an active aerobic respiration inside the colony biofilm caused oxygen to deplete in depth up to ca. $-200 \mu \mathrm{m}$, indicated by a decreasing air saturation. A profile 
measured immediately prior to removing the tobramycin (Figure 6B, profile b') shows that the air saturation increased up to $55 \%$ through the entire colony biofilm body (as oxygen penetrated all the way through the colony), and implies that the aerobic respiration zone moved from -200 $\mu \mathrm{m}$ depth (profile a') to the surface of the biofilm (equal to zero depth; profile b').

\section{Discussion}

We used an agar plate colony biofilm model to investigate the spatial distribution of aerobic respiration and denitrification activity in an ostensibly oxic in vitro environment, i.e., what might be expected in the upper and lower airways. Like other studies we found that aerobic respiration near the surface of the $P$. aeruginosa colony biofilm colony created an anoxic region in the interior ${ }^{6,25}$, in our case at below approximately $100 \mu \mathrm{m}$ depth. Bacterial denitrification in the lung was originally hypothesized on the basis of the CF lung being hypoxic ${ }^{8}$, due to increased epithelial oxygen consumption in combination with luminal hypoxia due to mucus plugging ${ }^{26}$; however as we, in line with others, show $P$. aeruginosa biofilms can create their own anoxic niches. On the addition of $\mathrm{NO}_{3}{ }^{-}$to the bulk liquid we observed an immediate response by the generation of $\mathrm{N}_{2} \mathrm{O}$. The rapid response suggested that the various enzymes involved in the denitrification steps were already expressed and functional allowing the cells to take immediate advantage of $\mathrm{NO}_{3}{ }^{-}$as a terminal electron acceptor. Surprisingly, we saw denitrification occurring even in the outer, oxic, layers of the colony biofilm demonstrating that, in the presence of $\mathrm{NO}_{3}{ }^{-}$, denitrification is possible even if the local environment is not hypoxic and the biofilm or biofilm aggregates are not large enough to create anoxic regions. Previously it was assumed that denitrification would only take place in the anoxic zones deep in the biofilm ${ }^{27}$. We used a concentration of $1.2 \mathrm{mM} \mathrm{NO}_{3}{ }^{-}$, which was relatively high compared to physiological concentrations, however concentrations of approximately $0.8 \mathrm{mM}$ have been reported in sputum from $\mathrm{CF}$ patients ${ }^{28}$, the pattern of denitrification distributed throughout the biofilm would be expected to be similar at lower concentrations. We found that although denitrification was occurring throughout the biofilm colony there was a high degree of variation at different depths in the biofilm (Fig. 4). This finding can be explained by a recent report using mRNA fluorescent in situ hybridization (FISH) reporters for metabolic genes for in situ single cell imaging which showed that in a $P$. aeruginosa biofilm there were single cells to pockets of cells approximately $25 \mu \mathrm{m}$ in diameter undergoing different metabolic pathways ${ }^{29}$. By direct measurement of denitrification within $P$. aeruginosa biofilm colonies biofilms our data supports the conclusions made by Hassett et al. ${ }^{3}$ and Yoon et al. ${ }^{7}$ that denitrification is occurring in the infected CF lung based on transcriptomic profiling.

With respect to antibiotic tolerance generally, it is assumed that part of the mechanism in $P$. aeruginosa biofilm infections, such as found in infected lung and chronic wounds, is due to inactivity within the biofilm due to nutritional limited dormancy. This hypothesis is based largely on experiments conducted in such a manner that nutrient conditions predilect for aerobic respiration. However, in the presence of $\mathrm{NO}_{3}{ }^{-}$, there is likely activity, albeit lower than that when metabolizing aerobically (i.e. Figure 5), all the way through the biofilm. After addition of tobramycin we observed an immediate reduction in aerobic 
respiration, but the microprofiles suggested it was never completely arrested. Furthermore, on removal of the tobramycin, the gradient began to steadily reestablish, suggesting that although there was inhibition there was no complete killing over the short exposure time of one hour. Recently it has been reported that phezanine, which is produced by $P$. aeruginosa, and can promote survival under anoxic conditions, can also provide tolerance to antibiotics ${ }^{6}$ which may explain our results.

\section{The agar colony biofilm model}

Our static biofilm model on agar plates immersed in MSM allowed the set-up of the microelectrodes to achieve simultaneous measurement of $\mathrm{DO}$ and $\mathrm{N}_{2} \mathrm{O}$ in close proximity in terms of location and depth in the biofilm was technically challenging. An advantage of using agar-grown colony biofilms was that we limited the probability of breaking the microelectrodes, which is a critical issue with biofilms grown on rigid surfaces such as glass. A disadvantage of using a soft material such as agar was that we could not detect the base of the colony biofilm with the applied sensors at the applied resolution and recording time, and unlike with a rigid surface, gradients developed at the biofilm-agar interface and into the agar as evidenced by the inflection point in $\mathrm{N}_{2} \mathrm{O}$ concentration at approximately $250 \mu \mathrm{m}$ depth as shown in Fig. 2. Another limitation of the model was that its complexity made it difficult to operate at $37^{\circ} \mathrm{C}$, which is more relevant when discussing our results in the context of infections. It is likely that the profiles would be similar, but conversion rates would be underestimated at room temperature, and DO would be less at $37^{\circ} \mathrm{C}$ than room temperature. Historically bacterial biofilms have been studied using experimental models in which biofilms are grown on rigid surfaces immersed in an aqueous solution containing nutrients for growth $^{30}$. However, increasingly soft substrate models are being developed to grow biofilms where biofilms are grown on hydrogel substrates under no, or low, shear to simulate growth on soft tissue such as might be found in an infected lung or chronic wound environment ${ }^{31}$. In some cases, the biofilms are grown directly on a hydrogel infused with nutrients ${ }^{31,32}$ or alternatively on a filter placed on the hydrogel as a "colony biofilm"33,34. These biofilms are grown under air rather than being immersed in a liquid. Colonies or lawns grown on agar plates are also increasingly being used as simple biofilm models for mechanical testing either after scraping the colonies off or directly on the colony itself ${ }^{35,36,37}$. While undoubtedly these colonies have high population densities there is limited information on how well they represent a "classical" biofilm phenotype. Recently Hoiby et al. ${ }^{38}$ presented evidence to suggest that $P$. aeruginosa spread as a lawn on an agar plate switched between a planktonic phenotype to a biofilm phenotype after between 5 to $7 \mathrm{hr}$ of incubation, evidenced by tolerance to antibiotics and production of the exopolysaccharide Psl in the extracellular polymeric substance (EPS) matrix. Schiessl et al. ${ }^{39}$ also reported ciprofloxacin tolerance in $P$. aeruginosa colony biofilms and related this to metabolic gradients using microelectrodes to show strong DO and redox gradients with an oxic region at the top of the colony and anoxic conditions at $\leq 50 \mu \mathrm{m}$ depth. Such gradients are caused by a combination of metabolic activity (consumption and production) and transport limitation into, out of and within the colony. They are well recognized as a characteristic of the biofilm phenotype and can explain phenomena such as the development of microenvironments and antibiotic tolerance through stationary phase-like dormancy to 
the build-up of cell signaling molecules for quorum sensing co-ordination of the population ${ }^{40}$. Although we recognize that colonies grown on an agar plate and then submerged in liquid differ markedly from conventional biofilms grown on solid surfaces under liquid, we found our model useful to demonstrate that in such colonies strong gradients develop as they do in other biofilms. And importantly that denitrification can occur even in the oxic upper layers of the biofilm simultaneously with aerobic respiration. It is unclear whether there may be pockets of cells in micro-anoxic niches within the oxic layer, which may explain/ have implications related to tolerance against antibiotics. Another unresolved aspect is if a small population of cells are denitrifying, despite having access to oxygen as a terminal electron acceptor, which may explain the occurrence of denitrifying 'persisters/ survivors' in the hypoxic environment that instantly switch on upon availability of oxidative nitrogen species. Nevertheless, our data reveal another aspect of metabolic versatility in $P$. aeruginosa biofilms that may help explain their persistence in chronic infections, and recalcitrance to antibiotic therapy.

\section{Declarations}

\section{AUTHORS AND CONTRIBUTORS}

PS and DdB originally conceived the experiments. PS, CUS and DdB designed and performed the experiments and performed data analysis. PS wrote the first draft of the manuscript and all authors contributed to editing subsequent versions.

\section{CONFLICTS OF INTEREST}

The authors declare that there are no conflicts of interest.

\section{FUNDING INFORMATION}

Hanse-Wissenschaftskolleg Institute for Advanced Study (DB and PS)

\section{ACKNOWLEDGEMENTS}

Thank you to Kendall Gallagher, Ohio State University for formatting.

\section{References}

1. Malhotra, S., Hayes, D., \& Wozniak, D.J. Cystic fibrosis and Pseudomonas aeruginosa: the host microbe interface. Clinical microbiology reviews 32(3), e00138-18, https://doi.org/10.1128/CMR.00138-18 (2019). 
2. Wu, Y.K., Cheng, N.C., \& Cheng, C.M. Biofilms in chronic wounds: pathogenesis and diagnosis. Trends in biotechnology 37(5), 505-517, https://doi.org/10.1016/j.tibtech.2018.10.011 (2019).

3. Hassett, D.J., Cuppoletti, J., Trapnell, B., Lymar, S.V., Rowe, J.J., et al. Anaerobic metabolism and quorum sensing by Pseudomonas aeruginosa biofilms in chronically infected cystic fibrosis airways: rethinking antibiotic treatment strategies and drug targets. Advanced drug delivery reviews 54(11), 1425-1443, https://doi.org/10.1016/S0169-409X(02)00152-7 (2002).

4. Line, L., Alhede, M., Kolpen, M., Kühl, M., Ciofu, O., et al. Physiological levels of nitrate support anoxic growth by denitrification of Pseudomonas aeruginosa at growth rates reported in cystic fibrosis lungs and sputum. Frontiers in microbiology 5, 554, https://doi.org/10.3389/fmicb.2014.00554 (2014).

5. Worlitzsch, D., Tarran, R., Ulrich, M., Schwab, U., Cekici, A., et al. Effects of reduced mucus oxygen concentration in airway Pseudomonas infections of cystic fibrosis patients. The Journal of clinical investigation 109(3), 317-325 https://doi.org/10.1172/JCl13870 (2002).

6. Schiessl, K.T., Hu, F., Jo, J., Nazia, S.Z., Wang, B., et al. Phenazine production promotes antibiotic tolerance and metabolic heterogeneity in Pseudomonas aeruginosa biofilms. Nature communications 10(1), 1-10 https://doi.org/10.1038/s41467-019-08733-w (2019).

7. Toyofuku, M. \& Yoon, S.S. Nitric oxide, an old molecule with noble functions in Pseudomonas aeruginosa biology. Advances in microbial physiology 72, 117-145 https://doi.org/10.1016/bs.ampbs.2018.01.005 (2018).

8. Yoon, S.S., Hennigan, R.F., Hilliard, G.M., Ochsner, U.A., Parvatiyar, K., et al. Pseudomonas aeruginosa anaerobic respiration in biofilms: relationships to cystic fibrosis pathogenesis. Developmental cell 3(4), 593-603 https://doi.org/10.1016/S1534-5807(02)00295-2 (2002).

9. Kamath, K.S., Krisp, C., Chick, J., Pascovici, D., Gygi S.P., et al. Pseudomonas aeruginosa proteome under hypoxic stress conditions mimicking the cystic fibrosis lung. Journal of proteome research 16(10), 3917-3928 https://doi.org/10.1021/acs.jproteome.7b00561 (2017).

10. Stewart, P.S., \& Franklin, M.J. Physiological heterogeneity in biofilms. Nature Reviews Microbiology 6(3), 199-210 https://doi.org/10.1038/nrmicro1838 (2008).

11. Ma, Y., Domingo-Felez, C., Plósz, B. G. \& Smets, B. F. Intermittent aeration suppresses nitrite-oxidizing bacteria in membrane-aerated biofilms: a model-based explanation. Environmental science \& technology 51, 6146-6155 (2017).

12. Stover, C., Pham, X., Erwin, A., Mizoguchi, S., Warrener, P., et al. Complete genome sequence of Pseudomonas aeruginosa PA01, an opportunistic pathogen. Nature 406(6799), 959-964 https://doi.org/10.1038/35023079 (2000).

13. Ohman, D.E., \& Chakrabarty, A. Genetic mapping of chromosomal determinants for the production of the exopolysaccharide alginate in a Pseudomonas aeruginosa cystic fibrosis isolate. Infection and immunity 33(1), 142-148 https://doi.org/10.1128/iai.33.1.142-148.1981 (1981).

14. Chan, R., Lam, J., Lam, K., \& Costerton, J.W. Influence of culture conditions on expression of the mucoid mode of growth of Pseudomonas aeruginosa. Journal of clinical microbiology 19(1), 8-16 
https://doi.org/10.1128/jcm.19.1.8-16.1984 (1984).

15. Gieseke, A., \& De Beer, D. Use of microelectrodes to measure in situ microbial activities in biofilms, sediments, and microbial mats. Molecular Microbial Ecology Manual 2, 1581-1612 (2004).

16. De Beer, D., Schramm, A., Santegoeds, C.M., \& Kuhl, M. A nitrite microsensor for profiling environmental biofilms. Applied Environmental Microbiology 63(3), 973-977 https://doi.org/10.1128/aem.63.3.973-977.1997 (1997).

17. Yoshinari, T., \& Knowles, R. Acetylene inhibition of nitrous oxide reduction by denitrifying bacteria. Biochemical and biophysical research communications 69(3), 705-710 https://doi.org/10.1016/0006-291X(76)90932-3 (1976).

18. Hancock, R.E.W., \& Speert, D.P. Antibiotic resistance in Pseudomonas aeruginosa: mechanisms and impact on treatment. Drug resistance updates 3(4), 247-255 https://doi.org/10.1054/drup.2000.0152 (2000).

19. von Ohle, C., Gieseke, A., Nistico, L., Decker, E.M., De Beer, D., et al. Real-time microsensor measurement of local metabolic activities in ex vivo dental biofilms exposed to sucrose and treated with chlorhexidine. Applied and environmental microbiology 76(7), 2326-2334 https://doi.org/10.1128/AEM.02090-09 (2010).

20. Schreiber, F., Stief, P., Gieseke, A., Heisterkamp, I.M., Verstraete, W., et al. Denitrification in human dental plaque. BMC biology 8(1), 1-11 https://doi.org/10.1186/1741-7007-8-24 (2010).

21. Versteeg, G.F., \& Van Swaaij, W.P. Solubility and diffusivity of acid gases (carbon dioxide, nitrous oxide) in aqueous alkanolamine solutions. Journal of Chemical \& Engineering Data 33(1), 29-34 (1988).

22. Yuan-Hui, L., \& Gregory, S. Diffusion of ions in sea water and in deep-sea sediments. Geochimica et cosmochimica acta 38(5), 703-714 https://doi.org/10.1016/0016-7037(74)90145-8 (1974).

23. Stewart, P.S. Diffusion in biofilms. Journal of bacteriology 185(5), 1485-1491 https://doi.org/10.1128/JB.185.5.1485-1491.2003 (2003).

24. Revsbech, N.P., \& Jørgensen, B.B. Microelectrodes: their use in microbial ecology. Advances in microbial ecology, 293-352 https://doi.org/10.1007/978-1-4757-0611-6_7 (1986).

25. Walters, M.C., Roe, F., Bugnicourt, A., Franklin, M.J., \& Stewart, P.S. Contributions of antibiotic penetration, oxygen limitation, and low metabolic activity to tolerance of Pseudomonas aeruginosa biofilms to ciprofloxacin and tobramycin. Antimicrobial agents and chemotherapy 47(1), 317-323 https://doi.org/10.1128/AAC.47.1.317-323.2003 (2003).

26. Montgomery, S.T., Mall, M.A., Kicic, A., \& Stick, S.M. Hypoxia and sterile inflammation in cystic fibrosis airways: mechanisms and potential therapies. European Respiratory Journal 49(1) https://doi.org.10.1183/13993003.00903-2016 (2017).

27. Stewart, P.S., White, B., Boegli, L., Hamerly, T., Williamson, K.S., Franklin, M.J., Bothner, B., James, G.A., Fisher, S., Vital-Lopez, F.G., \& Wallqvist, A. Conceptual model of biofilm antibiotic tolerance that integrates phenomena of diffusion, metabolism, gene expression, and physiology. Journal of bacteriology 201(22), e00307-19 https://doi.org/10.1128/JB.003017-19 (2019). 
28. Kolpen, M., Kühl, M., Bjarnsholt, T., Moser, C., Hansen, C.R., Liengaard, L., Kharazmi, A., Pressler, T., Høiby, N., \& Jensen, P.Ø. Nitrous oxide production in sputum from cystic fibrosis patients with chronic Pseudomonas aeruginosa lung infection. PLoS One 9(1), e84353

https://doi.org/10.1371/journal.pone.0084353 (2014).

29. Dar, D., Dar, N., Cai, L. \& Newman, D.K. Spatial transcriptomics of planktonic and sessile bacterial populations at single-cell resolution. Science 373(6556), eabi4882 https://doi.org/10.1126/science.abi4882 (2021).

30. Bahamondez-Canas, T.F., Heersema, L.A., \& Smyth, H.D.C. Current Status of In Vitro Models and Assays for Susceptibility Testing for Wound Biofilm Infections. Biomedicines 7(2), 34 https://doi.org/10.3390/biomedicines7020034 (2019).

31. Percival, S.L, Bowler, P.G., \& Dolman, J. Antimicrobial activity of silver-containing dressings on wound microorganisms using an in vitro biofilm model. International wound journal 4(2), 186-191 https://doi.org/10.1111/j.1742-481X.2007.00296.x (2007).

32. Ngo, Q.D., Vickery, K., \& Deva, A.K. The effect of topical negative pressure on wound biofilms using an in vitro wound model. Wound Repair and Regeneration 20(1), 83-90 https://doi.org/10.1111/j.1524475X.2011.00747.x (2012).

33. Folsom, J.P., Baker, B., \& Stewart, P.S. In vitro efficacy of bismuth thiols against biofilms formed by bacteria isolated from human chronic wounds. Journal of applied microbiology 111(4), 989-996 https://doi.org/10.1111/j.1365-2672.2011.05110.x (2011).

34. Hammond, A.A., Miller, K.G., Kruczek, C.J., Dertien, J., Colmer-Hamood, J.A., et al. An in vitro biofilm model to examine the effect of antibiotic ointments on biofilms produced by burn wound bacterial isolates. Burns 37(2), 312-321 https://doi.org/10.1016/j.burns.2010.09.017 (2011).

35. Kovach, K., Davis-Fields, M., Irie, Y., Jain, K., Doorwar, S., et al. Evolutionary adaptations of biofilms infecting cystic fibrosis lungs promote mechanical toughness by adjusting polysaccharide production. Npj Biofilms and Microbiomes 3(1), 1-9 https://doi.org/10.1038/s41522-016-0007-9 (2017).

36. Yannarell, S.M., Grandchamp, G.M., Chen, S.Y., Daniels, K.E., \& Shank, E.A. A dual-species biofilm with emergent mechanical and protective properties. Journal of bacteriology 201(18), e00670-00618 https://doi.org/10.1128/JB.00670-18 (2019).

37. Gloag, E.S., German, G.K., Stoodley, P., \& Wozniak, D.J. Viscoelastic properties of Pseudomonas aeruginosa variant biofilms. Scientific reports 8(1), 1-11 https://doi.org/10.1038/s41598-01828009-5 (2018).

38. Høiby, N., Henneberg, K.Å., Wang, H., Stavnsbjerg, C., Bjarnsholt, T., et al. Formation of Pseudomonas aeruginosa inhibition zone during tobramycin disk diffusion is due to transition from planktonic to biofilm mode of growth. International journal of antimicrobial agents 53(5), 564-573 https://doi.org/10.1016/j.ijantimicag,2018.12.015 (2019).

39. Schiessl, K.T., Hu, F., Jo, J., Nazia, S.Z., Wang, B., et al. Phenazine production promotes antibiotic tolerance and metabolic heterogeneity in Pseudomonas aeruginosa biofilms. Nature 
communications 10(1), 1-10 https://doi.org/10.1038/s41467-019-08733-w (2019).

40. Hall-Stoodley, L., Costerton, J.W., \& Stoodley, P. Bacterial biofilms: from the natural environment to infectious diseases. Nature reviews microbiology 2(2), 95-108 https://doi.org/10.1038/nrmicro821 (2004).

\section{Figures}

\section{Figure 1}

Colony thickness as function of time for PAO1 (open circles) and FRD1 (black circles). Mean and 1. STD of five measurements from different colonies on the same petri plate.

\section{Figure 2}

Experimental set-up for ex vivo agar plate colony biofilm growth modelling. A reservoir containing the petri plate with agar colony biofilms showing the positioning of the $\mathrm{DO}$ and $\mathrm{N}_{2} \mathrm{O}$ microelectrodes (left and right) and the reference electrode (center). B) Closer view from the side showing the electrodes in the reservoir. $C$ and D) The two microelectrodes just touching the surface of colonies grown on two different plates. The surface of the colony biofilm was identified when the shadows cast by the electrodes colocalized with the tips of the microelectrodes. An even flow of growth medium was supplied above the colonies by a pump (invisible).

\section{Figure 3}

DO $(A, B)$ and $\mathrm{N}_{2} \mathrm{O}(\mathrm{C}, \mathrm{D})$ profiles in PA01 and FRD1 agar colony biofilms measured in two different colonies ( $n=2)$ on the same petri plate (open and closed symbols) after the addition of $1.2 \mathrm{mM} \mathrm{NaNO}_{3}$. The bulk liquid-colony biofilm interface is presented by $0 \mu \mathrm{m}$ depth. While positive values present the colony biofilm body, negative values present the liquid phase. 


\section{Figure 4}

Local activity of aerobic respiration and denitrification (calculated from $\mathrm{NO}_{3}{ }^{-}$consumption based on $\mathrm{N}_{2} \mathrm{O}$ measurements) as a function of depth in PA01 and FRD1 colony biofilms measured in two different colonies on the same plate (light and dark bars) after the addition of $1.2 \mathrm{mM} \mathrm{NaNO}_{3}$. The bulk liquidcolony biofilm interface is represented at $0 \mu \mathrm{m}$ biofilm depth. Positive values on the $\mathrm{x}$-axis of $\mathrm{DO}$ and $\mathrm{NO}_{3}$ profiles show aerobic respiration and denitrification, respectively. Calculations are based on and correspond to the concentration profiles shown in Figure 3.

\section{Figure 5}

Local areal uptake rate of $\mathrm{DO}$ and $\mathrm{NO}_{3}{ }^{-}$measured for in PA01 and FRD1 colony biofilms on the same plate (light and dark bars indicate data from the different profiles) after the addition of $1.2 \mathrm{mM} \mathrm{NaNO}_{3}$.

\section{Figure 6}

Tobramycin, reduced aerobic respiration, but activity immediately recovers after its removal. A) Time trace of DO at $100 \mu \mathrm{m}$ depth in a PAO1 colony biofilm. Tobramycin was added at $1 \mathrm{hr}$ and removed at 1 $\mathrm{hr}$ after addition. Depth profiles were taken immediately prior to the time trace (a') and $1.5 \mathrm{hr}$ after antibiotic addition $\left(b^{\prime}\right)$. The gap in the time trace is where the second profile was taken. B) DO profiles measured prior to the start of the time trace (a', black circles) and $1.5 \mathrm{hr}$ after ( $b^{\prime}$ ', open circles) tobramycin addition.

\section{Supplementary Files}

This is a list of supplementary files associated with this preprint. Click to download.

- SuppFig1300DPI.tif

- Supp.Fig2300DPI.tif 